\title{
Reversible conduction block of peroneal nerve associated with SARS-CoV-2
}

\author{
Ettore Cioffi ${ }^{1}\left[\right.$ Davide Dilenola $^{1} \cdot$ Luigi luliano $^{1} \cdot$ Alessandro Polidoro $^{1} \cdot$ Carlo Casali $^{1} \cdot$ Mariano Serrao $^{1}$
}

Received: 7 June 2021 / Accepted: 6 October 2021 / Published online: 14 October 2021

(c) Fondazione Società Italiana di Neurologia 2021

\begin{abstract}
Background The ongoing SARS-CoV-2 pandemic, which is dramatically spreading worldwide, is well known for its respiratory sequelae. Besides cases of Guillain-Barré Syndrome, encephalitis, hyposmia, the whole range of neurological complications due to SARSCoV-2 is still not well known.

Methods and findings Herein, we report a new case of COVID-19, associated with mononeuropathy with reversible conduction block (CB). After SARS-CoV-2 infection, the patient developed acute weakness of left peroneal muscles. He underwent an endovenous immunoglobulin treatment, and symptoms improved. Two electroneurographic exam (before and after treatment), showed a reversible CB on left peroneal nerve. Dosage of serum antiganglioside antibodies showed anti-GM1 IgM positivity. Conclusions The present case gives new informations about reversible CB neuropathy as an acute presentation of SARS-CoV-2. Besides, antiganglioside antibodies evaluation could be useful to understand etiology of the increasing number of neurological manifestations related to SARS-CoV-2.
\end{abstract}

Keywords Neuroimmunology $\cdot$ Peripheral neuropathy $\cdot$ CIDP $\cdot$ Antiganglioside antibodies $\cdot$ SARS-CoV-2 $\cdot$ COVID-19

The ongoing SARS-CoV-2 pandemic, which is dramatically spreading worldwide, is well known for its respiratory sequelae [1]. Patients with COVID-19 disease typically present with severe viral pneumonia, often life-threatening, and other complications (gastrointestinal, renal, cardiovascular, endocrine, and hepatic systems can be hit) [2]. Neurological complications are also described: hyposmia, ageusia, headache, encephalitis, Guillain-Barré syndrome (GBS) [3].

Herein, we report a new case of COVID-19, associated with mononeuropathy with reversible conduction block (CB). On 11 November 2020, a 59-year-old man, presenting with fever $\left(\operatorname{Tmax} 38.7^{\circ} \mathrm{C}-101.66^{\circ} \mathrm{F}\right)$, dry cough, diarrhea, and asthenia, went to his family doctor, who sent him to the COVID-19 test center at our hospital in the suspect of SARS$\mathrm{CoV}-2$. He had no neurological sign or symptom. A real-time polymerase chain reaction (RT-PCR) nasopharyngeal swab (NPS) for SARS-CoV-2 was performed, resulting positive. Since the mild COVID-19-related symptoms, the family

Ettore Cioffi

ettore.cioffi@uniroma1.it

1 Department of Medico-Surgical Sciences and Biotechnologies, University of Rome Sapienza, Via Faggiana 34, 04100 Latina, Italy doctor decided to treat the patient at home, in quarantine regimen, and started a therapy with antibiotics, steroids, and low molecular weight heparin. The patient did not develop SARS-CoV-2 pneumonia, thus was not admitted to Intensive Care Unit (ICU). COVID-19-related symptoms lasted for 4 days. On 12 December 2020, after a new RT-PCR NPS for SARS-CoV-2 resulted negative, the patient came at our attention for clumsy gait with left steppage which appeared 12 days earlier (01 December 2020). After admission at our neurorehabilitation department, neurological examination disclosed weakness of the left anterior tibial, toe extensors, and peroneal muscles, with residual strength grade $3 / 5$ according to the Medical Research Council (MRC) scale. Laboratory results on admission were unremarkable. Cerebrospinal fluid (CSF)'s test showed cell count, protein, and glucose levels in normal range; RT-PCR assay for SARS-CoV-2 on CSF resulted negative. Screening tests for Epstein-Barr virus, Campylobacter jejuni, cytomegalovirus, Herpes viruses panel, Varicella zoster virus, influenza A virus, Haemophilus influenzae, HIV, and culture examinations were negative. On 17 December 2020 (T1), nerve conduction studies showed a CB of the left peroneal nerve at the popliteal fossa (Table 1; Fig. 1). Dosage of serum antiganglioside antibodies with indirect ELISA test 
Table 1 Values from the peroneal nerves' conduction studies both at $\mathrm{T} 1$ and T2. Values describing reversible conduction block are written in bold (decrease in amplitude - greater than 50\% - and CV recorded by the left peroneal nerve at $\mathrm{T} 1$, not evidenced at $\mathrm{T} 2$ ), compared with the healthy peroneal nerve

\begin{tabular}{|c|c|c|c|c|}
\hline & Distal latency & Amplitude & Fibular head-ankle CV & $\mathrm{F}$ waves mean \\
\hline \multirow[t]{3}{*}{ Left peroneal nerve (T1) } & \multirow[t]{3}{*}{$4.69 \mathrm{~ms}$} & Ankle $6.9 \mathrm{mV}$ & & \multirow[t]{3}{*}{$54.8 \mathrm{~ms}$} \\
\hline & & Fib head $5.7 \mathrm{mV}$ & Fib head - ankle $41 \mathrm{~m} / \mathrm{s}$ & \\
\hline & & Pop fossa $2.8 \mathrm{mV}$ & Pop fossa - fib head $29 \mathrm{~m} / \mathrm{s}$ & \\
\hline \multirow[t]{3}{*}{ Left peroneal nerve (T2) } & \multirow[t]{3}{*}{$3.75 \mathrm{~ms}$} & Ankle $7.4 \mathrm{mV}$ & & \multirow[t]{3}{*}{$51.9 \mathrm{~ms}$} \\
\hline & & Fib Head $6.3 \mathrm{mV}$ & Fib head - Ankle 45.5 m/s & \\
\hline & & Pop fossa $7.6 \mathrm{mV}$ & Pop fossa - Fib head $45.7 \mathrm{~m} / \mathrm{s}$ & \\
\hline \multirow[t]{3}{*}{ Right peroneal nerve (T1) } & \multirow[t]{3}{*}{$3.76 \mathrm{~ms}$} & Ankle $8.3 \mathrm{mV}$ & & \multirow[t]{3}{*}{$52.2 \mathrm{~ms}$} \\
\hline & & Fib head $8.1 \mathrm{mV}$ & Fib head - ankle 46 m/s & \\
\hline & & Pop fossa $8.9 \mathrm{mV}$ & Pop fossa - fib head $50 \mathrm{~m} / \mathrm{s}$ & \\
\hline \multirow[t]{3}{*}{ Right peroneal nerve (T2) } & \multirow[t]{3}{*}{$3.73 \mathrm{~ms}$} & Ankle $8.2 \mathrm{mV}$ & & \multirow[t]{3}{*}{$52 \mathrm{~ms}$} \\
\hline & & Fib head $8.2 \mathrm{mV}$ & Fib head - ankle $47.1 \mathrm{~m} / \mathrm{s}$ & \\
\hline & & Pop fossa $8.7 \mathrm{mV}$ & Pop fossa - fib head $49 \mathrm{~m} / \mathrm{s}$ & \\
\hline
\end{tabular}

Fig. 1 Amplitude $5 \mathrm{mV}$; duration $50 \mathrm{~ms}$. In red circles, a $50 \%$ increase in amplitude of distal compound muscle action potential at $\mathrm{T} 2$ (from 2.8 to $8.7 \mathrm{mV}$ ) can be seen
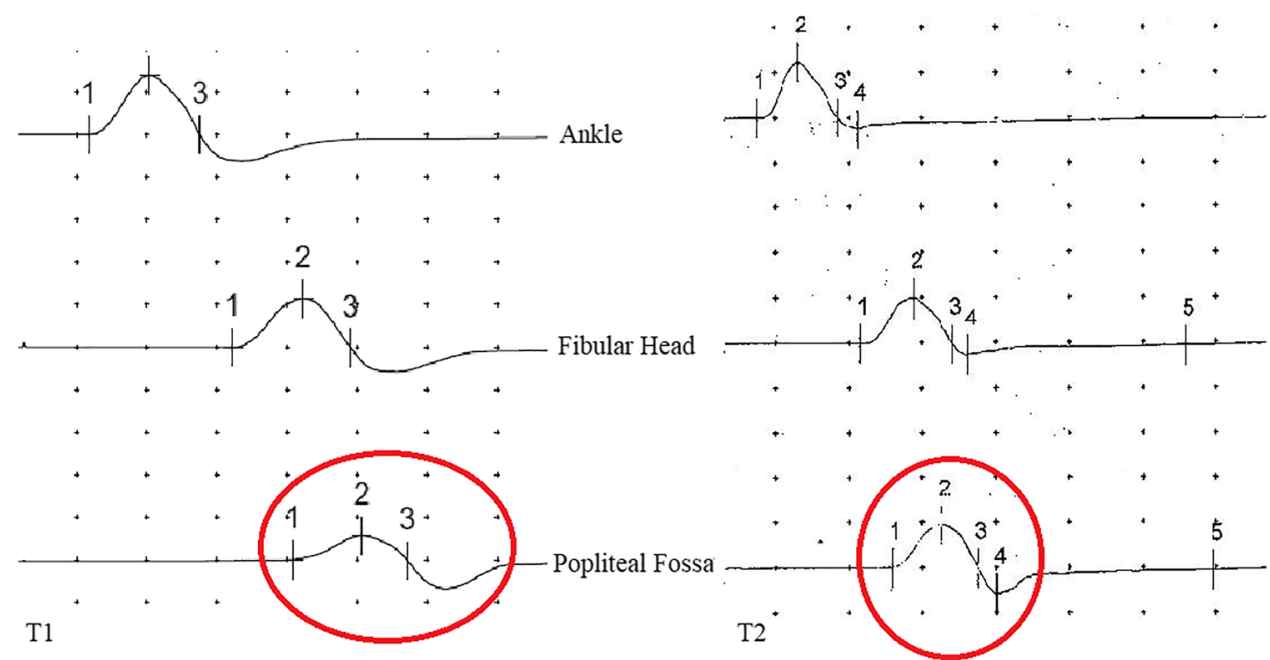

showed anti-GM1 IgM positivity (index 53.4; normal value 0-50).

Clinical, laboratory, and neurophysiological findings were consistent with mononeuropathy with reversible $\mathrm{CB}$ associated with anti-GM1 antibodies. However, we had to exclude other pathologic situations such as compressive causes, or Slimmer's paralysis [4]. These etiologies were unlikely, since the patient did not assume prolonged positions which could cause compression of peroneal nerve [5], nor had traumas, hematomas, or loss of weight (Slimmer's paralysis). Also, systemic causes of peroneal neuropathy were excluded since the normalcy of laboratory results. Thus, the patient was treated with a 5-day protocol of intravenous immunoglobulins (IVIg) at the dosage of $0.4 \mathrm{~g} / \mathrm{kg}$ / day, with subsequent improvement. On 03 February 2021, the patient underwent a follow-up neurophysiologic exam (T2), which was normal (Table 1; Fig. 1).
To date, several cases of SARS-CoV-2-associated GBS have been reported [6]; although, no other cases of SARSCoV-2 infection associated with mononeuropathy with reversible $\mathrm{CB}$ have been described. It is possible that the aforedescribed event may remain isolated, and a relapse may not occur, but also it could represent the onset of a disease belonging to the symmetric or asymmetric CIDPs' spectrum (such as multifocal motor neuropathy, or Lewis-Sumner neuropathy, or "classical" CIDP) [7], further considering the remarkable improvement with IVIg therapy. Although, we cannot totally exclude the possibility of spontaneous improvement. To be sure, regular clinical and neurophysiological follow-ups will need to be carried out, to define if the present case represents an isolated one, or any of the abovementioned relapsing-remitting dysimmune neuropathies. Seen the novelty of COVID-19 immunopathology, anti-GM1 antibodies may represent a new etiopathogenic mechanism underlining COVID19-related reversible CB 
neuropathy, although in our case we have mildly increased levels of antibodies, given their low level (i.e., 53.4; upper value 50). During SARS-CoV-2 disease, complexes of gangliosides are targeted by autoantibodies in GBS and its variants, being the GD1a/GD1b one of the most frequent [6]. Also, anosmia in COVID-19 could be explained by antiganglioside antibodies, seen the conspicuous expression of GD1a gangliosides in the olfactory bulb [8].

The present case gives a new information about reversible CB neuropathy as a possible acute presentation of SARSCoV-2. Besides, antiganglioside antibody evaluation could be useful to understand etiology of the increasing number of neurological manifestations related to SARS-CoV-2.

Author contribution Material preparation, data collection, and analysis were performed by Ettore Cioffi, Alessandro Polidoro, Luigi Iuliano, and Davide Dilenola. The first draft of the manuscript was written by Ettore Cioffi. All authors read and approved the final manuscript. Mariano Serrao and Carlo Casali supervised the final version of the manuscript. Ettore Cioffi and Davide Dilenola contributed equally to the paper.

Data availability Raw data and materials are available upon request.

\section{Declarations}

Ethical approval This study has been approved by our appropriate ethics committee (Ethical Committee of Sapienza University-Comitato Etico Lazio 2) and has been performed in accordance with the ethical standards laid down in the Declaration of Helsinki of 1964 and its later amendments.

\section{Conflict of interest None.}

Informed consent was obtained from the patient. Also, the patient signed informed consent regarding publishing its data.

\section{References}

1. Attaway AH, Scheraga RG, Bhimraj A, Biehl M, HatipoÄ Ÿlu U (2021) Severe COVID-19 pneumonia: pathogenesis and clinical management. BMJ 372:n436. https://doi.org/10.1136/bmj.n436

2. Mahajan RK, Paul G, Mahajan R, Gautam PL, Paul B (2020) Systemic manifestations of COVID-19. J Anaesthesiol Clin Pharmacol 36(4):435-442. https://doi.org/10.4103/joacp.JOACP_359_ 20

3. Whittaker A, Anson M, Harky A (2020) Neurological manifestations of COVID-19: a systematic review and current update. Acta Neurol Scand 142(1):14-22. https://doi.org/10.1111/ane.13266

4. Sotaniemi KA (1984) Slimmer's paralysis-peroneal neuropathy during weight reduction. J Neurol Neurosurg Psychiatry 47(5):564-566. https://doi.org/10.1136/jnnp.47.5.564

5. Chang LG, Zar S, Seidel B, Kurra A, Gitkind A (2021) COVID-19 proned ventilation and its possible association with foot drop: a case series. Cureus 13(4):e14374. https://doi.org/10.7759/cureus. 14374

6. Abu-Rumeileh S, Abdelhak A, Foschi M, Tumani H, Otto M (2021) Guillain-Barré syndrome spectrum associated with COVID-19: an up-to-date systematic review of 73 cases. J Neurol 268(4):1133-1170. https://doi.org/10.1007/s00415-020-10124-x

7. Lehmann HC, Burke D, Kuwabara S (2019) Chronic inflammatory demyelinating polyneuropathy: update on diagnosis, immunopathogenesis and treatment. J Neurol Neurosurg Psychiatry 90(9):981-987. https://doi.org/10.1136/jnnp-2019-320314

8. Cutillo G, Saariaho A-H, Meri S (2020) Physiology of gangliosides and the role of antiganglioside antibodies in human diseases. Cell Mol Immunol 17:313-322

Publisher's note Springer Nature remains neutral with regard to jurisdictional claims in published maps and institutional affiliations. 